Trauma Berufskrankh 2015 • [Suppl 2]:

17:315-321

DOI 10.1007/s10039-015-0005-9

Online publiziert: 14. Mai 2015

๑) Springer-Verlag Berlin Heidelberg 2015
Frank Schröter

Institut für Medizinische Begutachtung Kassel, Kassel, Deutschland

\section{Distorsion der Halswirbelsäule}

\section{Neue gutachtliche Aspekte}

Die Begutachtung von Unfallbeteiligten, die eine "Distorsion" der Halswirbelsäule (HWS) erlitten haben, wurde über Jahrzehnte hinweg geprägt von Unsicherheiten in der Diagnostik des primären Verletzungsbildes und der möglicherweise hieraus resultierenden gesundheitlich nachteiligen Folgen. Einer rationalen gutachtlichen Betrachtungsweise standen insbesondere die vielen vermeintlich beschwerdeerklärenden Hypothesen entgegen, die in den letzten Jahren durch zielgerichtete wissenschaftliche Untersuchungen weitestgehend aus der gutachtlichen Szene verschwunden sind. Es ist nicht zuletzt auch ein Verdienst der Rechtsprechung, mit einem Drängen auf die Beachtung beweisrechtlicher Grundregeln - z. B. den erforderlichen Vollbeweis des sog. Erstschadensbildes - für eine rationalere Betrachtungsweise in der Begutachtung gesorgt zu haben.

\section{Gesicherte Erkenntnisse}

Die einfache unfallmechanische Logik besagt, dass eine Verletzung geschützter Strukturen erst dann eintreten kann, wenn die schützenden Strukturen überwunden und damit geschädigt wurden.

Übertragen auf den Halsbereich bedeutet dies, dass beim wachen Fahrzeuginsassen die zur aufrechten Kopfführung notwendige muskuläre Anspannung mit Beteiligung zahlreicher Muskeln bewegungsdynamisch überwunden wird, da dann erst eine unkontrollierte Bewegung der HWS erfolgen kann. Eine „Distorsion“ der Strukturen der HWS selbst kann nur dann erfolgen, wenn die bewegungsdynamisch eingeleiteten Kräfte eine Bewegung der HWS über die Grenzen der physiologischen Bewegungsmöglichkeiten hinaus erzwingen und alternativ eine Bewegung in eine physiologisch nicht vorgesehene Richtung bewirken.

Jeder gutachtlich erfahrene Arzt weiß jedoch um die Effizienz dieses Muskelschutzes schon ausweislich der Beobachtung, dass eine passive Funktionsdiagnostik der HWS im Rahmen der Begutachtung bei willentlicher muskulärer Feststellung des Kopfes auch mit hohem Krafteinsatz des Untersuchers nicht möglich ist.

Da bei einem wachen Fahrzeuginsassen eine zusätzliche Muskelanspannung bei einer Fahrzeugkollision schon etwa $30 \mathrm{~ms}$ vor Beginn der bewegungsdynamisch induzierten Kopfbewegungen einsetzt [3], kann eine distorsionsbedingte HWS-Schädigung ohne vorherige Dehnungsbelastungen der schützenden Muskulatur nicht eintreten. Wenn somit über eine „HWS-Distorsion“ diskutiert wird, muss grundsätzlich hinterfragt werden, ob die klinischen Befundverhältnisse in der Frühphase nach dem Unfallgeschehen Indizien für eine muskuläre Beteiligung im Sinne der Zerrung erkennbar sind. Nach heutigem Erkenntnisstand bedarf es hierzu einer ganz erheblichen Bewegungsdynamik des Kopfes, wie sie bei leichteren, vorwiegend innerörtlichen Kollisionen schwerlich erreichbar ist.

Hieraus lassen sich drei gesicherte Erkenntnisse ableiten:

1. Fahrzeugkollisionen im niedrigen Geschwindigkeitsbereich bewirken beim wachen Fahrzeuginsassen keine Verletzung der HWS. Auch eine Muskelzerrung ist hierbei i.d.R. nicht zu erwarten.

2. Bei höheren Kollisionsgeschwindigkeiten sind Muskelzerrungen im Hals- und Nackenbereich möglich. Hiernach muss bei der Erstuntersuchung gezielt gefahndet werden, um das sog. Erstschadensbild zu sichern.
In Einzelfällen zeigt das Magnetresonanztomogramm (MRT) ein Muskelödem oder auch kleine umschriebene muskuläre Einblutungen.

3. Strukturelle Verletzungen an der HWS selbst sind selten, aber mittels MRT problemlos nachzuweisen, sodass dann auch die Begutachtung nicht auf ein gesichertes Erstschadensbild abgestellt werden kann.

Die Untersuchungen von Hinz [9] ergaben, dass bei Leichenversuchen und den dabei nota bene fehlenden muskulären Schutzmechanismen nur dann strukturelle Läsionen bewirkt werden konnten, wenn im betroffenen Segment Texturstörungen („degenerative“ Veränderungen) vorbestanden. Eine strukturell gesunde HWS ließ sich selbst mit sehr hohen Beschleunigungs- bzw. Entschleunigungsimpulsen nicht strukturell schädigen.

Die Überprüfungen nach Unfällen mit Beteiligung lebender Personen - Analyse von 64.000 Verkehrsunfällen - durch Hinz u. Plaue [10] ergaben, dass beim Lebenden mit vorbestehenden degenerativen Segmentveränderungen jedoch nur in seltenen Fällen strukturelle Läsionen nachzuweisen waren, was die weitreichende Suffizienz muskulärer Schutzmechanismen erkennen ließ.

\section{Ableitungen für die praktische Begutachtung}

Da jedermann in dem Istzustand versichert ist, wie er den Unfall erleidet, sich aber unterschiedliche versicherungsrechtliche Ableitungen aus diesem „Vorzustand" in den verschiedenen Rechtsbereichen ergeben, ist der Sachverständige gehalten, diesen Vorzustand anhand ana- 
mnestischer Daten und insbesondere der Bildbefunde zu objektivieren.

Die Feststellung einer vorbestehenden Texturstörung der Bandscheibenmatrix („Degeneration“) ist dabei für den Unfallbeteiligten keineswegs von nachteiliger Bedeutung: Besteht eine erhöhte Verletzungsbereitschaft (Vulnerabilität) im Bewegungssegment und bewirkt der Unfall nur deshalb eine strukturelle Schädigung, die bei einem zuvor anatomisch Gesunden nicht zu erwarten war, besteht besonders im Haftpflichtfall ein uneingeschränkter Entschädigungsanspruch, obwohl basierend auf den gesicherten wissenschaftlichen Erkenntnissen davon auszugehen ist, dass ohne solche vorbestehenden Veränderungen kein struktureller Verletzungseintritt zu erwarten war. Auch im Bereich der gesetzlichen Unfallversicherung bewirkt eine solche - vollbeweislich zu belegende - Schadensanlage, dass die beim Gesunden zur Herbeiführung einer Verletzung nicht ausreichenden Krafteinleitungen dennoch „rechtlich wesentlich" die Verletzung bewirkt haben und das eingetretene Schadensbild rechtlich gesehen als unfallbedingt ,verursacht" aufzufassen ist.

Nur im Bereich der privaten Unfallversicherung wird in einer solchen Fallgestaltung die Frage zu beantworten sein, in welcher prozentual zu bemessenden Höhe diese vorbestehende Schadensanlage mitverursachend war (,unfallfremde Mitwirkung"), was - nur - in diesem Versicherungsbereich zu einem Abzug bei der Versicherungsleistung führen kann.

\section{Verhalten des Unfallbeteiligten unmittelbar nach Unfall}

Wesentliche, für die gutachtliche Beurteilung relevante Informationen ergeben sich aus den Mitteilungen des Probanden, wie er den Zeitraum unmittelbar nach der Fahrzeugkollision in Erinnerung hat. Auch der Zeitpunkt des erstmaligen Wahrnehmens einer schmerzhaften Symptomatik ist für die Beurteilung von erheblicher Bedeutung.

Nach Verkehrsunfällen werden gar nicht selten auch von unverletzt gebliebenen Beteiligten Schreckreaktionen mit vielfältigsten Erscheinungsbildern - über Sekunden anhaltende Bewusstseinsein- trübung, subjektiv erlebte Parästhesien an Armen und Beinen, Zittern, Unfähigkeit zum geordneten Denken, Angstreaktionen, fast regelhaft verknüpft mit vegetativen Reaktionen wie Übelkeit, Schwindel und Kopfschmerz etc. - berichtet, die bei entsprechend veranlagten Personen so weit gehen können, dass sie unfähig sind, das Fahrzeug aus eigener Kraft zu verlassen, obwohl sie - wie sich später häufig herausstellt - körperlich unverletzt geblieben sind. Dies konnte sogar experimentell bei ca. $20 \%$ der freiwilligen Probanden mittels - nur vorgetäuschten - Crashversuchen nachgewiesen werden [7].

Dieser Personenkreis ist i.d.R. auch unfähig, gegenüber dem Gutachter die Unfallentstehung und den Unfallablauf näher zu beschreiben. Gerade diese stressinduzierten psychisch determinierten Beschwerden - laienhaft gerne als „Unfallschock" bezeichnet - führen leider immer wieder dazu, dass seitens der dann behandelnden Ärzte von einem „schweren Schleudertrauma“ gesprochen und damit die nachfolgende versicherungsrechtliche Auseinandersetzung bereits vorprogrammiert wird.

Häufig wird berichtet, dass nach einem kurzen Moment der Desorientiertheit die Situation überblickt wird, die betroffene Person eigentätig den Gurt löst und aussteigt, den Fahrzeugschaden in Augenschein nimmt und bei anderen Unfallbeteiligten sich nach möglicherweise eingetretenen Verletzungen erkundigt, also zielgerichtet handelt bis hin zur Verständigung der Polizei oder eines evtl. benötigten Kranken- oder Abschleppwagens. In diesen Fällen verbleibt der Unfallbeteiligte an der Unfallstelle, nimmt an der polizeilichen Unfallprotokollierung teil und setzt nicht selten mit dem eigenen beschädigten PKW - alternativ mit dem Abschleppfahrzeug - seine Fahrt fort, räumt dann in aller Regel auch ein, dass anfänglich gar keine subjektiv wahrnehmbaren Beschwerden bestanden haben.

Solche Angaben sind für die Feststellung des Eintritts einer Verletzung - auch der Schwere derselben - von erheblicher Bedeutung. Sie berühren insbesondere die Problematik des „,beschwerdefreien Intervalls".

Die Unterstellung eines beschwerdefreien Intervalls trotz eingetretener Verlet- zung kollidiert mit der ganz banalen Erfahrung, dass selbst harmlose Prellungen, Zerrungen oder Stauchungen nur mit einer Verzögerung von Sekunden Schmerzempfindungen zu bewirken pflegen, die sich dann stets relativ rasch, nämlich innerhalb von wenigen Minuten weitgehend verlieren und danach über wenige Tage hinweg nur dann noch bemerkt werden, wenn das gereizte Gewebe gedehnt wird („Bewegungsschmerz") oder eine Druckbelastung („Druckschmerz“) erfährt. Von Hinz u. Plaue [10] wurde nach Auswertung von 64.000 Unfällen vorgetragen, dass selbst bei leichteren und harmlosen Verletzungen eine Sofortsymptomatik zu erwarten ist, die i.d.R. auch von den betroffenen Personen wahrgenommen wird, lediglich der Höhepunkt des Schmerzerlebens - evtl. auch nach einem beschwerdearmen Intervall - zeitlich etwas nachhinkt, dies möglicherweise bis hin zum Unfallfolgetag, ehe dann diese Beschwerden nach und nach in einer sehr engen zeitlichen Beziehung zum Unfallgeschehen (binnen Tagen) auch wieder abzuklingen pflegen.

Handelt es sich um eine strukturelle Läsion (Fraktur, Zerreißung etc.), wird das primäre Schmerzerleben intensiver und anhaltender sein und insbesondere immer dann wieder nachhaltig aufleben, wenn die betroffene Struktur bewegt oder belastet wird. Systematische Untersuchungen haben ergeben, dass wache Unfallbeteiligte ausnahmslos nach Eintritt einer strukturellen HWS-Verletzung sofort über nachhaltige lokale Beschwerden im Halsbereich geklagt haben [16], sodass ein Ausbleiben solcher Sofortbeschwerden nach dem heutigen Stand der wissenschaftlichen Diskussion zumindest eine strukturelle Verletzung sicher ausschließt. Beobachtet wurden gelegentlich verzögerte Entwicklungen von Beschwerdeanteilen, z. B. die Entwicklung von Nervendruckerscheinungen durch sich langsam entwickelnde Weichteilödeme oder sogar Einblutungen im Bereich der Nervenaustrittsöffnungen an der HWS [10]. Auch Schluckbeschwerden können bei strukturellen Verletzungen am vorderen Längsband verzögert entstehen. Die zugrunde liegende strukturelle Verletzung muss jedoch stets zu einer Sofortsymptomatik führen. 
Die anamnestische Exploration offenbart gar nicht selten die Mitteilung, dass erst bei einem Kontakt mit Angehörigen oder am Arbeitsplatz von Unbeteiligten Bedenken geäußert werden, dass vielleicht doch eine Verletzung eingetreten sein könnte. Erst dann werden Beschwerden von der betroffenen Person subjektiv erlebt und führen zu einer ärztlichen Konsultation.

Ein solches, gelegentlich zeitraubendes anamnestisches Interview zu dieser Zeitphase zwischen Unfall und erstem ärztlichen Kontakt erscheint gutachtlich unverzichtbar, da hieraus bereits weitreichende Rückschlüsse gezogen werden können, die im Weiteren mit den ärztlichen Befundfeststellungen bei der Erstuntersuchung und im weiteren unfallnahen Zeitraum abzugleichen sind.

\section{Erstbefund}

Den ärztlicherseits erhobenen Erstbefunden kommt - zumindest unter beweisrechtlichen Aspekten, also aus juristischer Sicht - ein hoher Stellenwert zu, da das „Erstschadensbild“ der hohen Beweisqualität des „Vollbeweises“ unterliegt. Ist dieser Vollbeweis nicht zu erbringen, ist rechtstheoretisch von einer nichtverletzten Person auszugehen, sodass naturgemäß auch keine Folgen der Primärverletzung entstehen und verbleiben können. Folgt man also der reinen beweisrechtlichen Lehre, wäre bei fehlenden Befundmitteilungen in den Frühberichten das Prüfungsverfahren beendet. Die betroffene Person hat dann keine Aussichten mehr, irgendwelche Ansprüche geltend zu machen. So hat das Bundessozialgericht (BSG) im Urteil vom 07.09.2004 (Az.: B 2 U 25/03 R) zum „Beweisnotstand" festgestellt, dass bei Unaufklärbarkeit eines Umstandes, z. B. infolge insuffizient erhobener Erstbefunde, die Folgen der objektiven Beweislosigkeit dem zur Last fallen, der eine für ihn günstige Rechtslage geltend macht.

Das Erstschadensbild ist somit die entscheidende Brücke zwischen Unfalleinwirkung und später geäußerten Beschwerden und Symptomen. Allein der vollbeweislich belegte Unfall als solcher reicht hierfür nicht aus, da auch schwere Verkehrsunfälle gelegentlich unverletzt

Trauma Berufskrankh 2015 - [Suppl 2]: 17:315-321 DOI 10.1007/s10039-015-0005-9

(c) Springer-Verlag Berlin Heidelberg 2015

\section{F. Schröter}

\section{Distorsion der Halswirbelsäule. Neue gutachtliche Aspekte}

\section{Zusammenfassung}

Parallel zum stetig fortschreitenden wissenschaftlichen Erkenntniszugewinn unterliegen auch gutachtliche Algorithmen einem stetigen Wandel, zusätzlich noch mitgeprägt von einer fortlaufenden Weiterentwicklung der Rechtsprechung. Dies ist besonders deutlich spürbar bei der Begutachtung zum Thema "Distorsion der Halswirbelsäule", die in früheren Jahrzehnten geprägt war von der diagnostischen wie auch wissenschaftlichen Unsicherheit dessen, was sich hinter einer solchen Bezeichnung verbirgt. Infolge der durch die Magnetresonanztomographie wesentlich sicherer gewordene Diagnostik und durch die in die Bedeutungslosigkeit abgerutschten, vielfältigen hypothetisch-paramedizinischen
Lösungsansätze ist die Begutachtung zur Distorsion der HWS - weil nunmehr faktenorientiert - wesentlich rationaler, damit in der Aussage plausibler und nachvollziehbarer geworden. Ein strukturiertes Vorgehen in dem Prüfungsverfahren zur Kausalität ist eine der wesentlichsten Voraussetzungen für die „Annäherung an die Wahrheit durch Sorgfalt und Gewissenhaftigkeit bei Fertigung der Expertise." Hierfür steht der Sachverständige im Sinne der aristotelischen Tradition in der Pflicht.

\section{Schlüsselwörter}

Schleudertrauma $\cdot$ Hals $\cdot$ Begutachtung .

Kausalität $\cdot$ Rechtsprechung

\section{Distorsion of the cervical spine. New aspects of expert opinions}

\section{Abstract}

In parallel to the continuous gain and progression of scientific knowledge, assessment algorithms also undergo continual transformation and in addition are also influenced by successive further developments of the jurisdiction. This is especially perceptible in expert assessments on the topic of distorsion of the cervical spine which in previous decades was impacted by the diagnostic and scientific uncertainty, which underlies such a term. As a result of the substantially more certain diagnostics due to magnetic resonance imaging (MRI) and due to the slipping of the manifold hypothetical paramedical solution approaches into meaninglessness, assessment of distorsion of the cervical spine has become substantially more rational because it is factoriented and the conclusions have, therefore, become more plausible and comprehensible. A structured approach in the testing procedure on causality is one of the prime requirements for the "pursuit of the truth through diligence and conscientiousness when completing the expertise". In this context the expert is duty bound in the sense of the Aristotelian tradition.

\section{Keywords}

Whiplash injuries · Neck · Expert opinion . Causality · Jurisprudence von den Fahrzeuginsassen überstanden werden.

Insoweit ist es auch die wichtigste Aufgabe des Sachverständigen, diesen Vollbeweis für das Erstschadensbild zu führen. Hierauf wird jedoch in der übergroßen Zahl der gutachtlichen Prüfungen - zumindest wenn diese von gutachtlich unerfahrenen ärztlichen Kollegen erstellt werden - verzichtet, meist ersetzt durch die Feststellung, der Unfall sei aus Sicht des Gutachters zur Herbeiführung einer Verletzung im Hals- und HWS-Bereich "geeignet" gewesen mit der automatischen gedanklichen Verknüpfung der zum Untersuchungszeitpunkt noch geäußerten Beschwerden mit dem Unfallgeschehen.
Dabei wird übersehen, dass dieses "geeignet für" lediglich die Möglichkeit eines Verletzungseintritts umschreibt, jedoch nicht den Nachweis des tatsächlich eingetretenen Erstschadensbildes ersetzen kann.

Zur Feststellung des Erstschadensbildes genügt es auch nicht, wenn in einem knapp gefassten ärztlichen Attest nach der Erstuntersuchung lediglich die Diagnose einer „Distorsion“ oder eines „Schleudertraumas" der HWS nachzulesen ist. Solche ärztlichen Äußerungen beruhen - wie wissenschaftliche Untersuchungen ergeben haben - auf einer dramatisierend und damit den Arzt beeindruckenden Schilderung der Schwere des Unfallgeschehens, 
die beim Arzt Assoziationen zu einer dann „selbstverständlich“ zu erwartenden Verletzung weckt und unter forensischen Aspekten - der Arzt möchte sich nicht der Gefahr aussetzen, etwas übersehen zu haben - auch ohne entsprechende Befunde so gut wie regelhaft eine HWS-Distorsion bestätigt wird [14].

In sehr zahlreichen Erstbefunden ( $\mathrm{z}$. B. auch D-Arzt-Bericht) findet man nur globale Angaben über

- Muskelverspannungen, jedoch ohne genaue Lokalisationsangaben,

- Druckschmerzhaftigkeiten, meist polytop im gesamten Nacken und

- eine schmerzhafte Bewegungsminderung, global ohne Beschreibung der eingeschränkten Bewegungsrichtung und ohne Messdaten.

Diese sind jedoch in gleicher Weise bei einem unspezifischen Zervikalsyndrom anzutreffen, also nicht verletzungsbeweisend, noch nicht einmal verletzungsspezifisch oder auch nur verletzungstypisch [4, 12]. Mit einem Befundrapport, wie zuvor aufgelistet, kann der Sachverständige somit eigentlich gar nichts anfangen.

Bei einem tatsächlichen Verletzungseintritt und der dann stets zumindest begleitend vorliegenden Zerrung einzelner Muskelabschnitte im Halsbereich wird man im klinischen Bild - sofern sorgfältig untersucht wird - Folgendes erwarten dürfen [5]:

- Nur der gezerrte Muskel zeigt eine Tonuserhöhung und Druckdolenz.

- Nur diese Muskulatur entwickelt eine sog. Verkürzung.

- Nur hier ist ein Dehnungsschmerz auslösbar.

- Damit verknüpft ist eine asymmetrische Bewegungsstörung des Kopfes.

Solche differenzierten klinischen Befundmitteilungen sind jedoch in der Realität sehr selten.

Da nun in der Mehrzahl der Fälle die in der Frühphase gefertigten Bildbefunde nicht aussagekräftig sind, steht der Sachverständige bezüglich des von ihm abverlangten Vollbeweises für das Erstschadensbild häufig mit leeren Händen da. Die einzige Argumentation die bleibt, ist die Mitteilung des Probanden, vor dem Unfall beschwerdefrei gewesen zu sein, je- doch nicht mehr nach dem Unfall. Eine solche Behauptung - die prinzipiell nicht nachprüfbar ist - reicht jedoch zum objektiven Nachweis des Erstschadensbildes grundsätzlich nicht aus.

\section{Lösung des Kausalitätsproblems}

Selbstverständlich kann sich hinter einer solchen subjektiv mitgeteilten, aber eben unspezifischen Symptomatik auch eine unfallbedingt eingetretene Zerrung der Weichteile verbergen, die sich bildtechnisch dem notwendigen Nachweis entzieht. Eine hilfsweise und indirekte Beweisführung für einen solchen tatsächlichen - nichtstrukturellen - Verletzungseintritt ist dann nur noch mehrschrittig mittels Indizien zu führen:

- Keine Symptomatik im Zeitraum vor dem Unfallgeschehen

- Verletzungsmöglichkeit unfallmechanisch plausibel

- Beschwerdearmes Intervall längstens bis zum Morgen des Unfallfolgetages

- Passendes subjektives Beschwerdebild

- Beschwerdeprovokation an den Weichteilen durch Druck- und Bewegung im Bereich der gezerrten Areale

- Beschwerdemaximum im engen zeitlichen Verhältnis zur Unfalleinwirkung

- Abklingen der Beschwerden in einem überschaubaren Zeitraum von Tagen bis längstens 2 bis 3 Wochen

Dieser skizzierte Verlauf entspricht dem Regelfall einer nichtstrukturellen (Bagatell-)Verletzung entsprechend pathophysiologisch gesicherter Erkenntnisse zur Heilung, sodass mit diesen Verlaufsdaten die Kausalitätsfrage dahingehend beantwortet werden kann, dass es sich tatsächlich bei dem nach dem Unfallgeschehen beklagten Symptombild um eine Unfallfolge im Sinne der „funktionellen“ Läsion gehandelt hat.

Eine solche verlaufsorientierte Sicherung des Erstschadensbildes inklusive Kausalitätsbeurteilung schließt jedoch automatisch eine auf Dauer bestehende unfallbedingte Symptomatik aus, wie sie prinzipiell nach einer „funktionellen“ Verletzung unter pathophysiologischen Aspekten auch nicht erwartet werden kann.

\section{Bildgebende Diagnostik}

Es gilt inzwischen als unstreitig, dass die Nativröntgendiagnostik der HWS zum Nachweis einer HWS-Verletzung nur dann ein Ergebnis zeitigt, wenn eine strukturelle Läsion - insbesondere mit knöcherner Beteiligung - eingetreten ist.

Schon Erdmann [6] wie auch Kamith [11] zeigten auf, dass die "Steilstellung“ der HWS, häufig auch als „Streckfehlhaltung" bezeichnet, keine Indizwirkung für eine eingetretene Distorsion der Halswirbelsäule hat.

Zielgerichtete Untersuchungen [8] ergaben, dass eine Streckhaltung in der gesunden Bevölkerung in einer Größenordnung von $42 \%$ anzutreffen ist, bei Patienten mit chronischen Nackenschmerzen in $26 \%$ der Fälle, bei unfallbeteiligten Personen aber nur in $19 \%$.

Wenn man bedenkt, dass eine gute Röntgenassistentin bei Fertigung der Seitaufnahme stets zu einer möglichst gestreckt-aufrechten Kopfhaltung auffordert, um auch die untere HWS - befreit von Überlagerungseffekten der Schultern - darstellen zu können, erscheint die Argumentation, eine Steilstellung belege einen Verletzungseintritt, nur noch schlicht unsinnig.

Die von Arlen [1] inaugurierte biometrische Röntgenfunktionsdiagnostik erwies sich in den Folgejahren als so erheblich fehlerbelastet, auch die Normvarianz der Messwerte als so breit angelegt, dass hiermit keine Filterung funktionsgestörter - oder gar verletzter - Bewegungssegmente möglich war [15].

Zeigt das MRT in der Frühphase Ödembildungen an den Weichteilen, kann dies u. U. ein Indiz für eine stattgehabte Zerrung sein, wenn die Lokalisation des Ödems mit dem klinischen Befund (z. B. Druck- und Bewegungsschmerz) übereinstimmt.

Sofern umschriebene Einblutungen in der Muskulatur nachzuweisen sind, belegt dies eine, dann immer noch als harmlos zu bezeichnende mikrostrukturelle Läsion, die jedoch anders zu behandeln ist als eine reine Zerrung, die normalerweise keiner Behandlung - außer anfangs mit Analgetika - bedarf.

Eine technisch einwandfreie MRT offenbart mit relativ hoher Sicherheit eine 


\begin{tabular}{|c|c|c|c|c|c|c|}
\hline Verletzung & Klinik & MRT & Therapie & Dauer der AU & Dauerschaden & MdE \\
\hline $\begin{array}{l}\text { Keine } \\
\text { Grad } 0\end{array}$ & $\begin{array}{l}\text { Stressinduziert er- } \\
\text { höhter Muskeltonus }\end{array}$ & Leer & Entdramatisierung & 0 & 0 & 0 \\
\hline $\begin{array}{l}\text { Funktionell } \\
\text { Grad I }\end{array}$ & Nackensteifigkeit & $\begin{array}{l}\text { Ödem infolge } \\
\text { Weichteilzerrung }\end{array}$ & $\begin{array}{l}\text { Keine, evtl. temporär An- } \\
\text { algetika }\end{array}$ & 0 & 0 & 0 \\
\hline $\begin{array}{l}\text { Mikrostrukturell } \\
\text { Grad II }\end{array}$ & $\begin{array}{l}\text { Schmerzprovokation } \\
\text { durch Bewegung }\end{array}$ & $\begin{array}{l}\text { Einblutungen } \\
\text { infolge Faserrup- } \\
\text { turen }\end{array}$ & $\begin{array}{l}\text { Analgetika, evtl. physikali- } \\
\text { sche Therapie }\end{array}$ & 0 bis ca. 14 Tage & $\begin{array}{l}\text { Unwahr- } \\
\text { scheinlich }\end{array}$ & 0 bis $<10 \%$ \\
\hline $\begin{array}{l}\text { Makrostrukturell } \\
\text { (Grad III) }\end{array}$ & $\begin{array}{l}\text { Lokalisierter Schmerz } \\
\text { auch ohne Bewe- } \\
\text { gung, evtl. neurologi- } \\
\text { sche Symptomatik }\end{array}$ & $\begin{array}{l}\text { Strukturver- } \\
\text { letzung, "bone } \\
\text { bruise" }\end{array}$ & Befundorientiert & Nach Heilverlauf & \multicolumn{2}{|c|}{ Objektiver Befund maßgebend } \\
\hline
\end{tabular}

in seltenen Fällen eingetretene Strukturverletzung, nicht zuletzt durch das dann zu erwartende "bone bruise" in den knöchernen Strukturen, durch perifokale Ödembildungen oder gar Einblutungen im HSW-Bereich selbst.

Eine Bandscheibenvorwölbung (Protrusion) bis hin zum Prolaps ist immer schicksalhafter Natur und entspricht nicht dem typischen strukturellen Verletzungsbild im MRT, da der Nucleus prolaps selbst bei kompletter Faserringzerreißung - wie auch experimentell bestätigt [2] - nicht aus der Bandscheibe herauszutreten pflegt. Der Bandscheibenvorfall entspricht somit stets einem langzeitig abgelaufenen schicksalhaften Prozess.

Sofern infolge einer umfassenden Primärdiagnostik, inklusive MRT, feststeht, dass keine strukturelle Verletzung an evtl. vorbestehend texturgestörten Bewegungssegmenten eingetreten ist, verliert die Schadensanlage in der Kausalitätsbetrachtung ihre Bedeutung. Es ist dann allenfalls noch denkbar, dass solche vorbestehenden Veränderungen längerfristig beklagte Beschwerden im Sinne eines Zervikalsyndroms bewirken. Eine solche Feststellung ist aber nur dann erlaubt, wenn neben dem radiologischen Befund auch ein entsprechender klinischer Segmentbefund in der radiologisch veränderten Segmenthöhe festzustellen ist. In der Mehrzahl der Fälle sind radiologisch detektierte texturgestörte Segmente nicht beschwerdeursächlich.

Die MRT hat sich in den letzten Jahren als ein ganz wesentliches Diagnostikum nach bewegungsinduzierten Verletzungen im Halsbereich etablieren können. Das Verfahren erlaubt die siche- re Feststellung einer nicht eingetretenen HWS-Verletzung wie auch einer eingetretenen Läsion und unterstützt maßgeblich eine befundorientierte Schweregradeinteilung (•Tab. 1) auf vollbeweislich gesicherter Befundbasis.

Die von Erdmann im Jahre 1973 inaugurierte Graduierung [6], die sich fast ausschließlich auf patientenseitige Subjektivismen stützt, entspricht nicht mehr den Ansprüchen der modernen Medizin. Moderne Schweregradeinteilungen von Verletzungen beruhen so gut wie ausnahmslos auf objektiven Befundkriterien. Die Ergebnisse der bildgebenden Verfahren haben dabei einen herausragenden Stellenwert erlangt. Es ist an der Zeit, dass diese modernen analytischen Denkweisen auch das Denken und Handeln der Ärzte bestimmen, die mit der Problematik der „HWS-Distorsion“ - insbesondere gutachtlich - konfrontiert werden.

Da die Schweregradeinteilung der Quebec Task Force [16] sich ebenso in ihrer Grundstruktur auf semiobjektive Befunde und insbesondere auch auf subjektive Beschwerden stützt, ist auch diese Klassifikation nicht unproblematisch, zumindest für gutachtliche Zwecke nicht tauglich.

Eine neu strukturierte Schweregradeinteilung, die sich überwiegend auf belastbare objektive Befundkriterien stützt, ist längst überfällig, um die Diskussion zur HWS-Distorsion und ihre Folgen in rationale Bahnen zu lenken (• Tab. 1).

\section{Gutachtliche Systematik}

In der medizinischen Begutachtung gilt der Grundsatz, dass der „Regelfall“ kei- ner besonderen Beweisführung bedarf, während ein Abweichen vom Regelfall den Sachverständigen zwingt, die pathophysiologische Plausibilität für diesen besonderen Fall herauszuarbeiten, um Überzeugungen zu vermitteln. Zu berücksichtigen sind dabei auch eingetretene Komplikationen oder Fehlbehandlungen, die - wenn die Behandlung auf Unfallfolgen ausgerichtet war - mit ihren Folgeerscheinungen auch dem Unfall zuzurechnen sind.

Geht der unfallchirurgische/orthopädische Gutachter systematisch in der Reihenfolge der hier besprochenen Prüfaspekte vor, wird er gemäß des Prüfschemas (•Abb. 1) Schritt für Schritt zu einer plausiblen gutachtlichen Beurteilung gelangen.

Erkennt er dabei Hinweise auf eine psychogene Symptomatik, endet jedoch die Beurteilungskompetenz des organmedizinisch orientierten Sachverständigen. Die in der Untersuchungssituation beobachteten Verhaltensauffälligkeiten, wie z. B. berichtete Ängste beim Autofahren, besonders beim Passieren der Unfallstelle, Tränenausbrüche bei Schilderung des Unfallgeschehens und organpathologisch nicht erklärbare Symptomschilderungen sollten nachdenklich stimmen und Veranlassung geben, eine zusätzliche nervenärztliche Begutachtung zu empfehlen.

\section{Gutachtliche Fehler}

Der häufigste gutachtliche Fehler liegt in der Behauptung, dass ein Unfall - dann meist angelehnt an die Erdmann-Graduierung -,„geeignet“ gewesen sei zur Verursachung einer Verletzung. Die aus der 


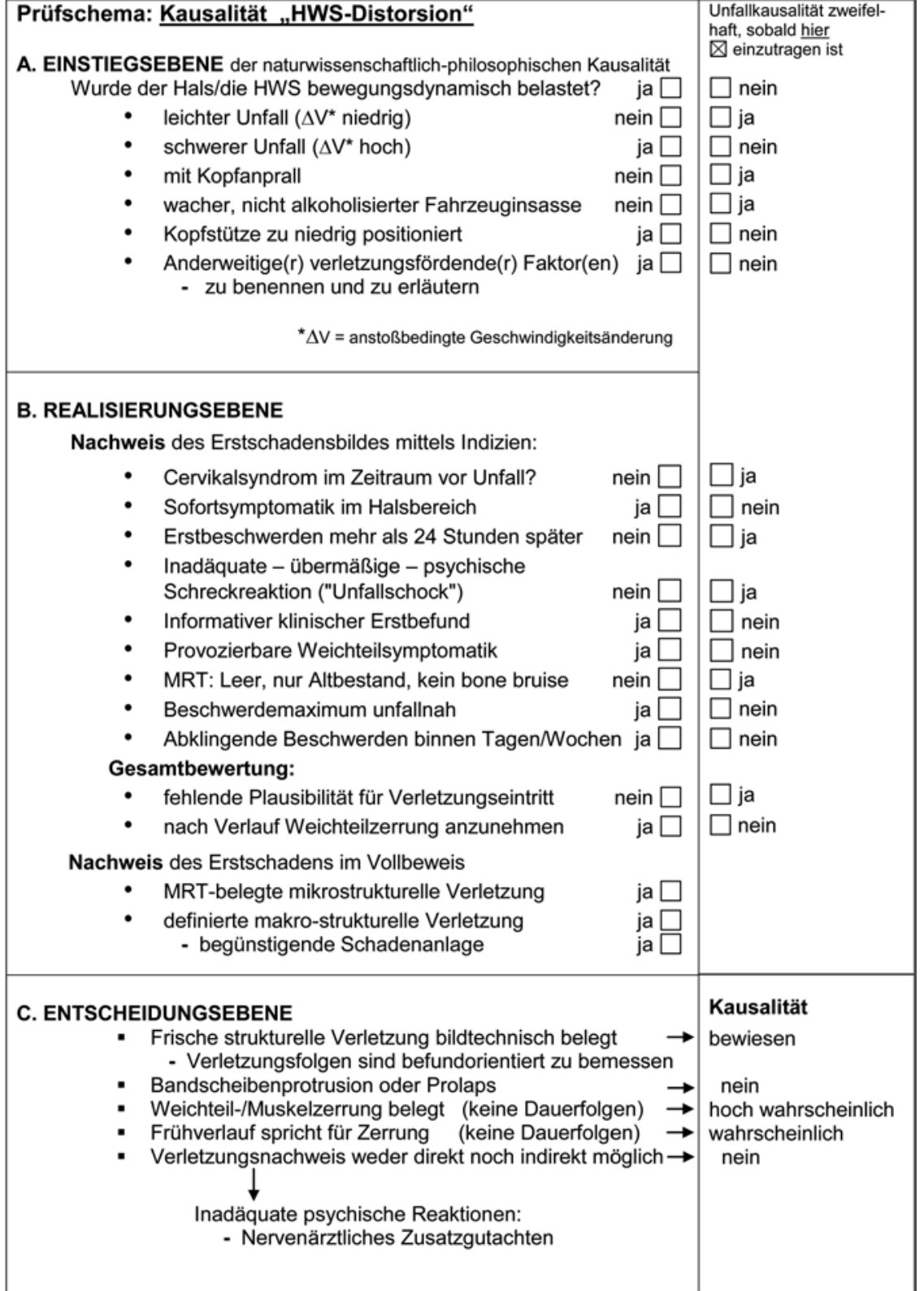

Abb. $1 \Delta$ Prüfschema zur Kausalität der HWS-Distorsion

Eignung abgeleitete Verletzungsmöglichkeit wird gleichgesetzt mit dem tatsächlichen Verletzungseintritt. Nicht selten wird aus der anhaltenden Dauer der Beschwerden ohne jegliche Prüfung anhand anderer Kriterien unter Berufung auf Erdmann [6] auf ein „schweres“ Schleudertrauma zurückgeschlossen. Der Sachverständige verzichtet dabei auf die Ausleuchtung der Anfangsbefunde und damit auf den Nachweis des Erstschadensbildes anhand belastbarer klinischer Befunddaten. Dieser eigentlich schlimmste Fehler in einem Gutachten wird dennoch nicht immer vom Auftraggeber bemerkt, auch nicht von den Gerichten.

Ein weiterer häufig zu beobachtender Fehler liegt darin, dass man der Einfachheit halber Beschwerden, die nach dem Unfall beklagt wurden, automatisch gleichsetzt als hervorgerufen durch den Unfall, was dem Prinzip des ,post hoc, ergo propter hoc“ entspricht. In der juristischen Gutachtenliteratur wird dies als dummer gutachtlicher Fehler bezeichnet [13]. Eine solche zeitliche Abfolge ist zwar die Eingangsvoraussetzung für einen Kausalzusammenhang, da die umgekehrte Reihenfolge den Kausalzusammenhang automatisch ausschließen würde. Sie ist jedoch nicht beweisend für eine Kausalitätsverknüpfung.

Als banaler, nicht dem Anspruch eines professionellen Gutachtens entsprechenden Fehler hat die Äußerung „... ich bin der Meinung, dass ..." zu gelten. Persönliche Meinungsäußerungen sind im Gutachten nicht gefragt, sondern ein $\mathrm{Ab}$ gleich der Fakten des konkreten Einzelfalls mit gesicherten wissenschaftlichen Erkenntnissen. Der Sachverständige, der so argumentiert, verzichtet regelhaft auf Darlegungen zu einer pathophysiologischen Plausibilität für eine Verknüpfung des Erstschadensbildes mit einer prolongierten Symptomatik. Eine solche Verknüpfung kann bei funktionellen Verletzungen nicht gelingen. Das anerzogene „Helfersyndrom“ des kurativ tätigen Arztes führt jedoch nicht selten da$\mathrm{zu}$, dennoch dem klagenden Patienten zuzustimmen.

Solche inadäquaten ärztlichen Begutachtungen dürften in den vergangenen Jahren und Jahrzehnten in Verbindung mit einer nachhaltigen juristischen Hilfestellung unter der Überschrift „HWSSchleudertrauma" zu einer in der Summe gewaltigen, durch eine verunsicherte Rechtsprechung sanktionierten - aber ungerechtfertigten - Schmerzensgeldleistung geführt haben.

\section{Korrespondenzadresse}

\section{Dr. F. Schröter}

Institut für Medizinische Begutachtung Kassel Landgraf-Karl-Str. 21, 34131 Kassel

f.schroeter@imb-kassel.de

\section{Einhaltung ethischer Richtlinien}

Interessenkonflikt. F. Schröter gibt an, dass kein Interessenkonflikt besteht.

Dieser Beitrag beinhaltet keine Studien an Menschen oder Tieren.

The supplement containing this article is not sponsored by industry.

\section{Literatur}

1. Arlen A (1979) Röntgenologische Röntgenfunktionsanalyse der HWS. Manuelle Med 17:24

2. Brinckmann P, Porter RW (1994) A laboratory model of lumbar disc protrusion. Spine 19:228-235 
3. Castro WHM, Schilgen M, Meyer S, Meyer S, Weber M, Peuker C, Wörtler K, Wörtler K (1997) Do „whiplash injuries" occur in low speed rear impacts? Eur Spine J 6:366-375

4. Domes M (2009) Das „HWS-Schleudertrauma“ aus gutachterlicher Sicht. Orthop Rheuma 1-2: 19

5. Dvorak J (1996) HWS-Verletzungen, Untersuchungskriterien, Stand der Therapie. Schweiz Z Soz Vers Berufl Vorsorge 40:453-461

6. Erdmann H (1973) Schleuderverletzung der Halswirbelsäule. Die Wirbelsäule in Forschung und Praxis, Bd 56. Hippokrates, Stuttgart

7. Hein MF et al (2000) Gibt es ein "HWS-Schleudertrauma" ohne biomechanische Belastung? Ergebnisse einer interdisziplinären Studie. Osteologie 9:36

8. Helliwell BS, Stevans BF, Wright V (1994) The straight cervical spine. Does it indicate muscle spasme? J Bone Joint Surg 76-B:103-106

9. Hinz P (1970) Die Verletzung der Halswirbelsäule durch Schleuderung und durch Abknickung. Die Wirbelsäule in Forschung und Praxis, Bd 47. Hippokrates, Stuttgart

10. Hinz P, Plaue R (1972) Die Begutachtung von Schleuder- und Abknickverletzungen der Halswirbelsäule. Akt Orthop 4:1-20

11. Kamieth $H$ (1986) Röntgenfunktionsdiagnostik der Halswirbelsäule. Die Wirbelsäule in For-schung und Praxis, Bd 105. Hippokrates, Stuttgart

12. Nordin M et al (2008) Assessment of neck pain and its associated disorders. Spine 22(4 Suppl):S101S122

13. Schneider E (1995) Logik für Juristen. Franz Vahlen, München

14. Schnabel M, Weber M, Vassiliou T, Mann D, Kirschner M, Gotzen L, Kaluza G (2004) Diagnostik und Therapie akuter Beschwerden nach "HWS-Distorsion" in Deutschland. Unfallchirurg 107:300-306

15. Schöps P, Siebert U, Schmitz U, Friedle AU, Beyer A (2000) Reliabilität nicht invasiver diagnostischer Untersuchungsmethoden zur Erfassung schmerzhafter Halswirbelsäulensyndrome. Manuelle Med 38:17-32

16. Spitzer WO et al (1995) Scientific monograph of the Quebec Task Force on whiplash-associated disorders. Spine (Phila Pa 1976) 20(Suppl VIII):85

17. Velmahos GC, Theodoru D, Tatevossian $R$ et al (1996) Radiographic cervical spine evaluation in the alert asymptomatic blunt trauma victim much ado about nothing? J Trauma 46:768-774 